\title{
CLIMATIC FACTORS AND EPIDEMIOLOGIC CHARACTERISTICS OF HEAD AND NECK SKIN MALIGNANCIES IN OSIJEK BARANJA COUNTY, CROATIA
}

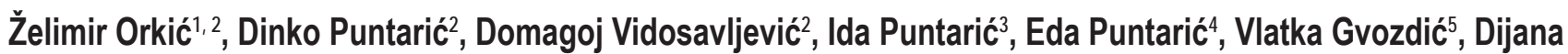 \\ Mayer ${ }^{6}$, Marina Vidosavljević ${ }^{7}$, Andrijana Muller Vranješ ${ }^{2}$ \\ ${ }^{1}$ Department of Surgery, University Hospital Centre Osijek, Osijek, Croatia \\ ${ }^{2}$ Faculty Of Medicine, J. J. Strossmayer University of Osijek, Osijek, Croatia \\ ${ }^{3}$ Institute for Emergency Medicine of Zagreb County, Velika Gorica, Croatia \\ ${ }^{4}$ Biology Department, Faculty of Science, Zagreb, Croatia \\ ${ }^{5}$ Department of Chemistry, J. J. Strossmayer University of Osijek, Osijek, Croatia \\ ${ }^{6}$ Croatian Institute for Public Health, Zagreb, Croatia \\ ${ }^{7}$ County General Hospital, Vinkovci, Croatia
}

\section{SUMMARY}

Aim: The aim of this study was to investigate and compare the incidence and epidemiological characteristics of head and/or neck skin malignancies in Osijek-Baranja County, Croatia, and to connect them with relative climatic indicators such as the number and intensity of sunny and cloudy days over a longer period of time.

Methods: The study included patients from Osijek Baranja County with confirmed pathohistological diagnosis of the head and/or neck malignancies from January 1, 2004 to December 31, 2012. The patients were analyzed according to gender, age, residence, occupation, type and location of the neoplasm, and hair and eye colour. The analysis of climatic elements (mean monthly and annual cloudiness values, mean monthly and annual sum values of sunny days) for a period of 50 years (1961-2011) based on the data of the Croatian Meteorological and Hydrological Service for the investigated area was performed.

Results: The study included a total of 2,952 patients: 1,487 (50.4\%) males and 1,465 (49.6\%) females, representing the estimated annual incidence of 104/100,000. The mean age was 72 years. The patients were mostly from rural areas, 1,952 (66.2\%); 2,137 (72.4\%) worked outdoors: 907 farmers (42.4\%) and 889 construction workers (41.6\%). Given the type of neoplasm, the most common was a basal cell cancer (BCC) in 2,160 patients (73.2\%). Malignant melanoma was found in 93 patients (3.1\%). The most common localizations were face (839 cases, $28.7 \%$ ) and nose (643 cases, 22.0\%). Males had a significantly higher number of planocellular malignancies - 341 (56.6\%) than females - 262 (43.4\%) $(p=0.005)$. The median age of the patients was 67 years. There were no significant differences in types of malignancies, place of residence, workplace, or occupation (with respect to working outdoors or indoors). It has been shown that the ears and lips are significantly more affected by squamous cell malignancies $(p=0.039$ and $p<0.001)$ compared to the neck, eye and head with malignant melanoma $(p=0.004, p<0.001$, and $p=0.026)$ and the nose where basal cell neoplasms $(p=0.002)$ prevail. There were no significant differences in the type and frequency of malignant neoplasms in relation to hair and eye colour of the patients. The last 50 years in Osijek-Baranja County have seen a declining trend in the number of cloudy days and upward trend in the mean annual sum of sunny days. When compared, the increase in sunny days results in a higher number of patients suffering from malignant melanoma $(\rho=0.695, p=0.038)$.

Conclusion: It is obvious that malignant neoplasms of the skin, head and neck occur after decades of exposure, and as a cumulative effect of exposure to risk factors. A direct exposure to sun seems to play an important role, especially with regard to melanoma. Nevertheless, further research is needed.

Key words: malignant neoplasms of the skin, head and neck, epidemiology, intensity of solar radiation

Address for correspondence: D. Vidosavljević, Faculty of Medicine, J. J. Strossmayer University of Osijek, Huttlerova 4, 31000 Osijek, Croatia. E-mail: domagoj.vidosavljevic@gmail.com

\section{INTRODUCTION}

\section{Climatic Factors and Carcinogenic Effects of Ultra- violet Radiation}

In the last hundred years the greatest warming of the Earth's surface over the last 1,300 years has been observed. Thus, between
1906 and 2006, the average temperature rose by $0.6-0.9^{\circ} \mathrm{C}$, and the rate of temperature increase has nearly doubled in the past 50 years. Long and short term changes in the intensity of solar heat radiation have had an impact on climate change (1). Thinning of the ozone layer, called the ozone "hole" is primarily a result of human activities in industry and transport. It is calculated that a $1 \%$ reduction in ozone increases UV radiation by $2 \%$ (2). Differ- 
ent molecules absorb greenhouse gases or different wavelengths of sunlight. For example, molecules of oxygen $\mathrm{O}_{2}$ and ozone $\mathrm{O}_{3}$ absorb almost all the sun's rays shorter than $320 \mathrm{~nm}$ in the ultraviolet part of the spectrum (UVB and UVC). In the spectrum of solar radiation, ultraviolet radiation accounts for only $10 \%$ of energy. The intensity of ultraviolet radiation and its composition depends on many factors including the length of the route through the atmosphere, which depends on the season, time of a day, latitude, altitude, cloudiness, and air purity (3).

The danger of UV radiation varies throughout the year. The most important influence on the intensity of sunlight at the Earth's surface is the height of the sun (season), altitude, clouds, reflections on the ground, and atmospheric ozone. UVB ultraviolet radiation level depends on the angle of solar light: it is lower in the winter, in the morning and late afternoon, and higher during the summer and at noon. Based on a series of measurements, weather reduces UV radiation in the following percentages: a clear sky allows $100 \%$ of UV radiation to pass through; high, thin clouds 89\%; broken clouds 73\%; and heavy clouds allow $31 \%$ of UV radiation to pass through (4). Thus, UV radiation is stronger when the sky is cloudless. Most natural surfaces, grass, soil and water reflect less than $10 \%$ of the radiation while snow can reflect up to $80 \%$ of the radiation. UV rays penetrate into the water and at a depth of $1 / 2$ meter the level of radiation from the surface is $40 \%$. When considering its effect on human health and the environment, ultraviolet radiation is usually classified into UVA (400-315 nm) or longwave (black light), UVB (315-280 nm) or medium and UVC $(<280$ $\mathrm{nm}$ ) or short (antimicrobial). Ultraviolet photons damage DNA molecules in a living organism in various ways. According to current knowledge, UVB radiation has the most powerful carcinogenic effect. Nucleic acids and proteins absorb the light of UVB region with the highest impact at 260 and $280 \mathrm{~nm}$. The absorption causes damage to the cellular DNA, which, if not repaired by a particular repair mechanism, can cause mutations that may result in malignant skin tumours (5). The skin has the ability to protect itself from the negative effects of UV radiation with specific protective mechanisms. These are pigmentation, thickening of the cornual layer of the skin, DNA repair mechanisms, and activation of the body's own antioxidants. If the skin is exposed to sunlight for too long, the skin photoprotection is insufficient and the DNA repair mechanisms become overloaded $(6,7)$. The possible harmful effects of solar radiation on the human skin should be taken into account when it comes to the types of ultraviolet radiation UVA and UVB. UVA and UVB rays penetrate through the outer layer of the skin and cause damage, e.g., burns, skin cancer, allergies etc. So UVB ultraviolet radiation, which does not penetrate into the deeper layers of the skin, causes acute damage to the skin (erythema) in the form of burns, which leads to the degeneration of the skin, aging and can cause skin cancer (8). The harmful effects of UV rays can be acute and chronic. Acute effects are sunburn, phototoxic and photoallergic reactions (various deodorants, perfumes). Acute idiopathic actions and actinic prurigo may also occur. One of the chronic effects of UV radiation is photoaging, which begins at birth. In Germany, 17 clinical studies analysing medical data arrived to conclusion that the effectiveness of protective means against solar radiation for the prevention of melanoma has not yet been fully tested (9).

\section{Skin Cancer of the Head and Neck}

The solar effect on the human body is cumulative, and the harmful effects of excessive sun exposure (especially sustained burns) in childhood and adolescence reflect at older age. This fact is supported by the higher incidence of malignant neoplasms of the skin in areas with stronger insolation and among people who are exposed to UV radiation for professional and recreational reasons $(9,10)$. Malignant neoplasm of the skin is the most common malignancy in the United States, with over 3.5 million new cases reported annually. Melanoma represents $3-5 \%$ of all malignant neoplasms of the skin, but it is responsible for the vast majority of deaths (11). Solar keratosis is the most common precancerosis and it is estimated that at least $65 \%$ of planocelluar and $36 \%$ of all baseocellular carcinoma develop from these skin lesions $(10,11)$.

Croatia, like many other countries, lack reliable data on the incidence of malignant neoplasms of the skin, particularly on the mortality from certain types of tumors, with a slightly better understanding of the situation regarding malignant melanoma (9). As a Central European and Mediterranean country with a large number of sunny days, but still with an insufficient level of awareness of the danger of sun exposure, Croatia is expected to have a higher frequency of these types of tumours in the future (12). A similar problem is present in other countries with a significant proportion of the white population, especially those with fair complexion, blond or red hair (and light coloured eyes) (7, 13-15). The aim of the study was to determine if there is a connection between meteorological conditions in terms of a smaller number of cloudy and increased number of sunny days and the number of skin tumours, and if it can be associated with a higher risk of tumour formation on uncovered areas of the skin.

Based on the available information about patients, possible differences in the morbidity due to age and gender, place of residence (urban and rural), different direct exposure to the sun (outdoor occupations) and reduced sunlight exposure (indoor occupations) will be determined.

\section{MATERIALS AND METHODS}

\section{Patients}

The study included patients treated at the Clinical Hospital Osijek from January 1, 2004 to December 31, 2012, with pathohistological confirmation of malignant neoplasms of the skin on the head and/or neck, all of them from Osijek-Baranja County. The patients were grouped according to diagnosis as follows: squamous cell carcinoma, basal cell carcinoma, squamous cell and basal cell carcinoma, and malignant melanoma. The study was approved by the Ethics Committee of the University Hospital Osijek.

\section{Methods}

The patients whose findings and history data were used in this study were classified into two groups based on the place of residence: city (urban areas) or village (rural areas). Each of these groups (urban and rural) was further divided according to gender and age. Regarding the exposure to sunlight, the patients were classified into two subgroups: those working outdoors (direct 
exposure to the sun) and indoors (less exposure to direct sunlight). The patients were also divided into three categories: farmers, other occupations exposed to direct sunlight (e.g. construction workers) and indoor occupations. The colour of hair and eyes was also recorded for some patients.

\section{Analysis of Climate Data}

An analysis of climatic elements (mean monthly and annual cloudiness values, mean monthly and annual sum of sunny days) for a period of 50 years (1962-2012) was made on the basis of the data obtained from the Croatian Meteorological and Hydrological Service. Time series data (sunshine, sunny or cloudy days, the mean daily cloudiness) are shown together with a floating fiveyear average for the year and the associated linear trend for the given period. Normality of distribution was tested using the Kolmogorov Smirnov test. Mean values of continuous variables are expressed by median and range for variables that do not distribute normally. Nominal figures are shown by frequency distribution per group and share distribution. For determination of the difference between two independent samples the Mann-Whitney U-test for nonparametric distribution was used, and the Kruskal Wallis test for three or more independent samples. To determine the differences between the proportions, $\chi^{2}$ - test and Fisher's exact test were used. Connection was determined by the Spearman's correlation coefficient. For assessment of the increase/decrease of frequency of the observed types of malignant neoplasms per year the Cochran-Armitage trend test for proportions was used. The data were analyzed using the SPSS 15.0 software package (SPSS Inc., Chicago, IL, USA) with a significance level of $\mathrm{p}=0.05$.

\section{RESULTS}

Osijek-Baranja County is located in northeast Croatia and covers an area of $4,152 \mathrm{~km}^{2}$ and is the fourth largest county in Croatia. The position of the county is $45^{\circ} 32^{\prime}$ ' north and $18^{\circ} 44^{\prime}$ east. According to the census of 2001, it had 330,506 inhabitants $(171,829$ women, 51.8\%; and 158,677 men, 48.2\%) (31), while in 2011, it had 305,032 inhabitants, but the proportion of women and men remained the same $(51.8 \%, 48.2 \%$, respectively) $(16,17)$. During nine years, 2,952 patients suffering from malignant neoplasms of the skin of the head and/or neck were recorded; 1,487 (50.4\%) men and 1,465 (49.6\%) women. Distribution by gender and age showed a significant difference (Fisher's exact test, $\mathrm{p}=0.022$ ). The average annual incidence for the entire follow-up period was approximately 104/100,000 ranging from 91/100,000 in 2004 to approximately 123/100,000 in 2012 with a growth tendency. The mean age of patients was 72 years (interquartile range 64-78 years). However, there were significantly younger participants in 2006, with a mean age of 69 years (interquartile range 60-75 years) (Kruskal Wallis test, $\mathrm{p}<0.001$ ). The patients were mostly from rural areas, 1,952 (66.2\%), with no significant difference during the studied years. Outdoor occupations included 2,137 patients (72.4\%) and indoor occupations 813 patients (27.6\%), (Fisher's exact test, $\mathrm{p}=0.003$ ). The most common among the outdoor occupations were farmers - 907 (42.4\%) and construction workers - 889 (41.6\%), whereas among indoor occupations there were factory workers - 204 (25.1\%), with significant differences according to age distribution (Fisher's exact test, $\mathrm{p}<0.001$ ) (Table 1). Given the type of neoplasm, 2,160 patients (73.2\%) had basal cell malignant neoplasm. Squamous cell carcinoma was diagnosed in 603 patients (20.4\%), whereas basal and squamous cell carcinoma type in 95 patients (3.2\%). Malignant melanoma was diagnosed in 93 patients (3.1\%), with the highest significance in 2010 (Fisher's exact test, $\mathrm{p}<0.001$ ). The most common localisations were the face - in 839 patients (28.7\%) and nose - in 643 patients (22.0\%). There was a significant difference among patients according to age ranging from 23 (7\%) in 2007 to 42 (11\%) in 2012, except for neoplasms that were located on the ear, where the number of patients was equal (Table 2).

In relation to the malignant neoplasm, gender, the only significant difference $(p=0.005)$ was for planocellular neoplasm. From a total of 603 cases, 341 were men (56.6\%) compared to 262 women (43.4\%) (Fisher's exact test, $\mathrm{p}=0.005$ ). Significantly, the youngest patients were the ones with malignant melanoma, with the median age of 67 years (interquartile range 57-71 years). There is no significant difference concerning the type of malignant neoplasms and place of residence, workplace and occupation (indoor, outdoor) (Table 3). The Fisher's exact test has shown that according to localization the ear and lip had significantly more squamous cell malignant neoplasms ( $p=0.039$ and $p<0.001$ ), while the neck, eye and head malignant melanoma ( $p=0.004, p<0.001$, and $p=0.026)$, and the nose basal cell neoplasms $(\mathrm{p}<0.001)$ (Table 4). Data on hair colour were collected in 1,717 cases, on eyes in 616 cases, but there were no significant differences in the type and frequency of malignant neoplasm in relation to hair and eye colour of the patients (Table 5).

By using the Spearman's correlation coefficient $(\rho)$ the connection between the number of patients with malignant neoplasms of the skin, head and neck and gender, place of residence, age, location, and the type of malignant neoplasm and the number of hours of sunshine was evaluated. It was established that an increase in the number of sun hours results in a higher incidence of malignant melanoma, while it does not affect the number of cases of other types of tumours $(\rho=0.695, p=0.038)$ (Table 6).

\section{Climatic Factors and Elements from 1962-2012 in Osijek-Baranja County}

The climate of Osijek-Baranja County is determined by a mixture of influences of the Eurasian mainland, the Atlantic Ocean and the Mediterranean Sea. According to Koeppen classification, it is a moderately warm, rainy climate, without significant dry periods, with rainfall evenly distributed throughout the year. The average temperature is 11 degrees Celsius $\left(11^{\circ} \mathrm{C}\right.$ spring, summer $21^{\circ} \mathrm{C}, 11.8^{\circ} \mathrm{C}$ autumn, winter $0.2^{\circ} \mathrm{C}$ ). The air is very humid, and the fall and winter with a lot of fog. This climate is particularly favourable for the growth of plants and the life of people and animals. Based on the presentation of sunshine duration (lasting irradiation by direct solar radiation and measured by heliograph) the mean duration of sunshine over 50 years has been 1,737 hours (interquartile range from 8.5 to 1,953) per year, and the iddle floating five-year average of 1,781 hours of sunshine (Fig. 1). The duration of sunshine (insolation) during 50 years at the measuring station of the city of Osijek indicates that from 1992 to 2011 insolation increased repeatedly to values above 2,000 hours per year. The mean deviation from the floating five-year average 


\begin{tabular}{|c|c|c|c|c|c|c|c|c|c|c|c|c|c|c|c|c|c|c|c|c|c|c|c|c|c|c|}
\hline & $=$ & & ฏ̊ & & & & $\begin{array}{l}\vec{b} \\
\text { o. } \\
\text { v }\end{array}$ & & $\begin{array}{l}\text { 苂 } \\
\text { 品 }\end{array}$ & & & $\begin{array}{l}\text { cok } \\
\stackrel{c}{c}\end{array}$ & & & & & ôt & & & & & & ثِّ & & & \\
\hline & 嵒 & & 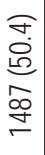 & 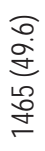 & 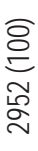 & & $\begin{array}{l}\mathbf{a} \\
\frac{0}{1} \\
\frac{d}{d} \\
\mathbb{N}\end{array}$ & & 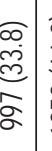 & ה్ & & 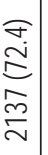 & $\begin{array}{l}\stackrel{0}{a} \\
\stackrel{d}{d}\end{array}$ & & 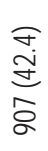 & 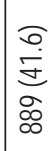 & 疍 & $\frac{\Theta}{\stackrel{\rho}{\rho}}$ & & $\begin{array}{c}\underset{m}{e} \\
\vec{e} \\
\overrightarrow{i n}\end{array}$ & 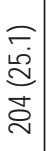 & 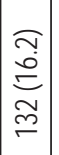 & $\begin{array}{l}\widehat{c} \\
\infty \\
\infty \\
\infty \\
0\end{array}$ & 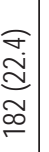 & $\begin{array}{l}\widehat{0} \\
\stackrel{0}{G} \\
\stackrel{9}{\infty} \\
\infty\end{array}$ & 胥 \\
\hline & సี่ & & 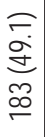 & 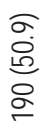 & 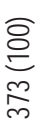 & & 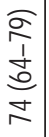 & & 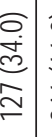 & 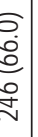 & & 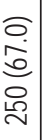 & 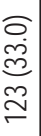 & & 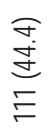 & 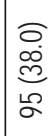 & 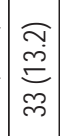 & $\underset{F}{\stackrel{F}{J}}$ & & $\underset{\sim}{\stackrel{f}{+j}}$ & 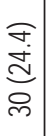 & 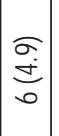 & 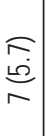 & 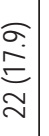 & 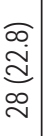 & $\begin{array}{l}\tilde{m} \\
\stackrel{d}{d} \\
\stackrel{2}{\sim}\end{array}$ \\
\hline & 고ํ & & 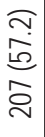 & 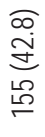 & $\begin{array}{l}\text { ठ্] } \\
\text { ్ㅠ }\end{array}$ & & 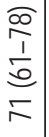 & & 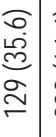 & 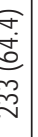 & & 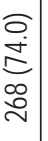 & 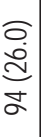 & & 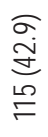 & 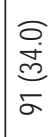 & $\begin{array}{l}\vec{f} \\
\stackrel{\sigma}{G} \\
\vec{f}\end{array}$ & 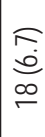 & & $\begin{array}{c}\sqrt{5} \\
\infty \\
\infty \\
\infty\end{array}$ & 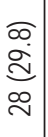 & 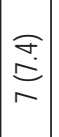 & $\begin{array}{c}\widehat{P} \\
\underset{f}{+}\end{array}$ & 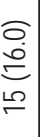 & 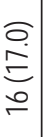 & 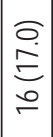 \\
\hline & 옴 & & 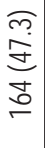 & 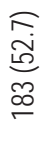 & 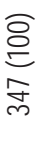 & & 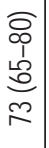 & & 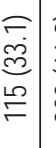 & 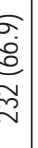 & & 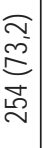 & 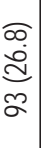 & & 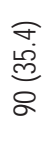 & 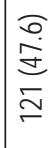 & 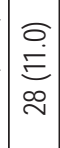 & 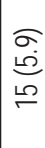 & & 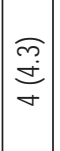 & 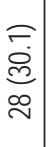 & 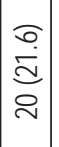 & 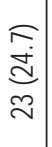 & 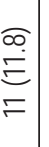 & 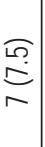 & 0 \\
\hline & : & & 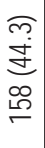 & 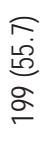 & 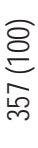 & & 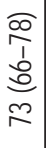 & & 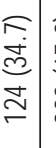 & שֶ. & & 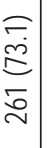 & $\begin{array}{l}\text { ब. } \\
\stackrel{d}{\mathbb{d}} \\
\stackrel{8}{8}\end{array}$ & & 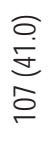 & 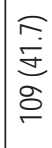 & 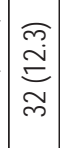 & $\begin{array}{l}\stackrel{\partial}{\rho} \\
\stackrel{\rho}{\rightarrow} \\
\end{array}$ & & $\begin{array}{l}\overline{0} \\
\infty \\
\infty \\
\infty\end{array}$ & 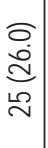 & 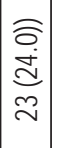 & $\begin{array}{l}\text { Fे } \\
\stackrel{0}{0} \\
\sigma\end{array}$ & 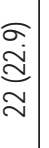 & $\underset{\substack{\mathcal{g} \\
\underset{\sigma}{f}}}{ }$ & $\stackrel{\Theta}{\omega}$ \\
\hline & : ర్ & & 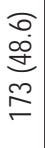 & 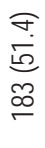 & 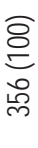 & & 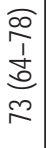 & & 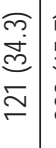 & ֶָ, & & 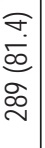 & 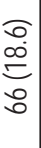 & & 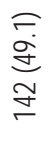 & $\begin{array}{l}\widetilde{\sigma} \\
\tilde{\tilde{\sigma}} \\
\tilde{\sigma} \\
\tilde{\sigma}\end{array}$ & 吕 & 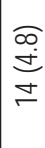 & & 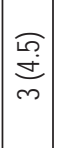 & $\begin{array}{l}\overparen{0} \\
\stackrel{0}{0} \\
\stackrel{d}{g} \\
\stackrel{g}{G}\end{array}$ & 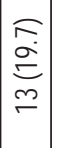 & 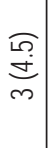 & & 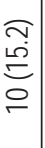 & $\begin{array}{l}\underset{ت}{ت} \\
\underset{\infty}{3}\end{array}$ \\
\hline & స్తి & & 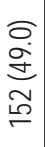 & 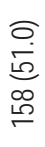 & $\begin{array}{l}\text { 일 } \\
\text { 일 }\end{array}$ & & 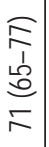 & & 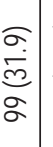 & ב. & & 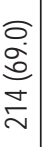 & $\begin{array}{l}\vec{\sigma} \\
\vec{i} \\
\vec{c} \\
\stackrel{8}{8}\end{array}$ & & $\begin{array}{l}\stackrel{7}{\mathcal{J}} \\
\stackrel{8}{8}\end{array}$ & 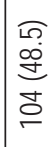 & $\begin{array}{l}\sigma \\
\rho \\
\rho \\
\sigma\end{array}$ & 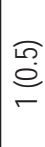 & & $\begin{array}{l}\text { ָ̦ } \\
\stackrel{N}{\sim}\end{array}$ & $\begin{array}{l}\widehat{\sigma} \\
\stackrel{0}{\vec{d}} \\
\stackrel{\vec{\sigma}}{9}\end{array}$ & $\mid \begin{array}{c}\tilde{m} \\
\infty \\
\infty \\
\infty\end{array}$ & 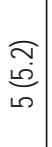 & 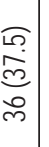 & $\underset{\sim}{\stackrel{\overrightarrow{\mathrm{d}}}{\sim}}$ & 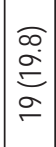 \\
\hline & ఫ্ & & 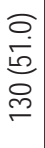 & 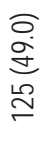 & 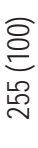 & & $\begin{array}{l}\widetilde{\rho} \\
\hat{1} \\
0 \\
8 \\
0\end{array}$ & & $\begin{array}{l}\widetilde{\sigma} \\
\text { o. } \\
\stackrel{0}{0} \\
\delta \\
\delta\end{array}$ & 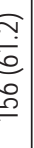 & & 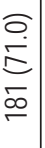 & $\begin{array}{l}\text { Oे } \\
\text { d } \\
\text { I }\end{array}$ & & 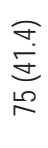 & 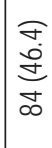 & $\underset{\stackrel{\sigma}{\sigma}}{\stackrel{\sigma}{\vec{d}}}$ & $\underset{-1}{\stackrel{\varrho}{\varrho}}$ & & $\begin{array}{l}\underset{\sigma}{\omega} \\
\dot{v} \\
\forall\end{array}$ & $\begin{array}{l}\stackrel{\sigma}{d} \\
\stackrel{\infty}{\vec{d}} \\
\vec{y}\end{array}$ & 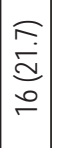 & $\underset{m}{\stackrel{\vec{d}}{+}}$ & 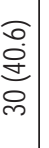 & $\underset{\sim}{\stackrel{\widetilde{d}}{\sim}}$ & $\begin{array}{l}0 \\
0 \\
0 \\
\omega\end{array}$ \\
\hline & 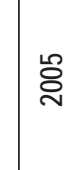 & & 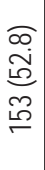 & 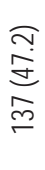 & 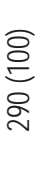 & & 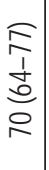 & & 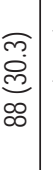 & 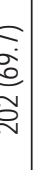 & & 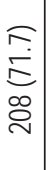 & 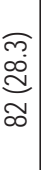 & & 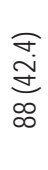 & 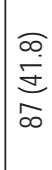 & 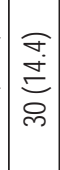 & $\underset{\mathrm{m}}{\stackrel{\text { I }}{\mathrm{i}}}$ & & $\begin{array}{l}\stackrel{\Omega}{\Omega} \\
\infty \\
\sim \\
\sim\end{array}$ & $\begin{array}{l}\tilde{\alpha} \\
\dot{d} \\
\tilde{\sim} \\
\approx\end{array}$ & 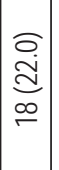 & $\begin{array}{c}\text { ָ̦ } \\
\stackrel{5}{0}\end{array}$ & 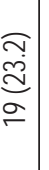 & 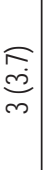 & 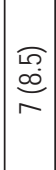 \\
\hline & চ্் & & 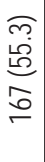 & 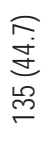 & 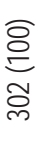 & & 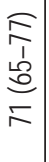 & & 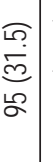 & 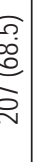 & & 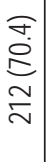 & $\begin{array}{l}\text { ర్ర } \\
\stackrel{\mathbb{d}}{\mathbb{D}} \\
\stackrel{8}{\infty}\end{array}$ & & 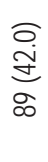 & 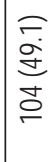 & 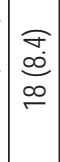 & 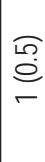 & & 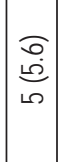 & 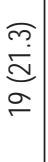 & $\begin{array}{l}\sigma \\
\stackrel{\tilde{d}}{d} \\
-\vec{N}\end{array}$ & 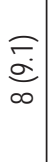 & $\begin{array}{c}\overrightarrow{7} \\
\stackrel{3}{\overrightarrow{3}} \\
\overrightarrow{7}\end{array}$ & 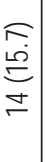 & $\begin{array}{l}\sigma \\
\omega \\
\omega \\
\omega \\
\omega\end{array}$ \\
\hline & & $\begin{array}{l}\bar{a} \\
z \\
\bar{d} \\
\bar{d} \bar{d} \\
0\end{array}$ & $\frac{10}{e^{\frac{\pi}{2}}}$ & 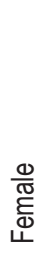 & त & & 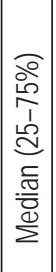 & 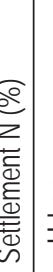 & 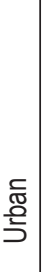 & & 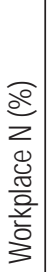 & $\begin{array}{l}\frac{\omega}{0} \\
\text { o. } \\
\overline{0} \\
0\end{array}$ & 응 & 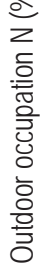 & 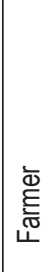 & 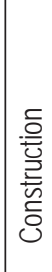 & 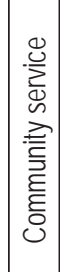 & $\begin{array}{l}\bar{\Phi} \\
\stackrel{\bar{\nu}}{0}\end{array}$ & 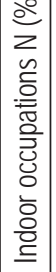 & 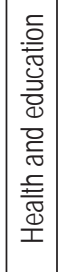 & 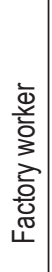 & 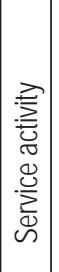 & 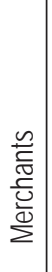 & 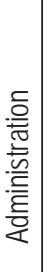 & 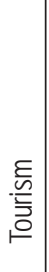 & 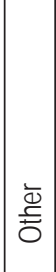 \\
\hline
\end{tabular}




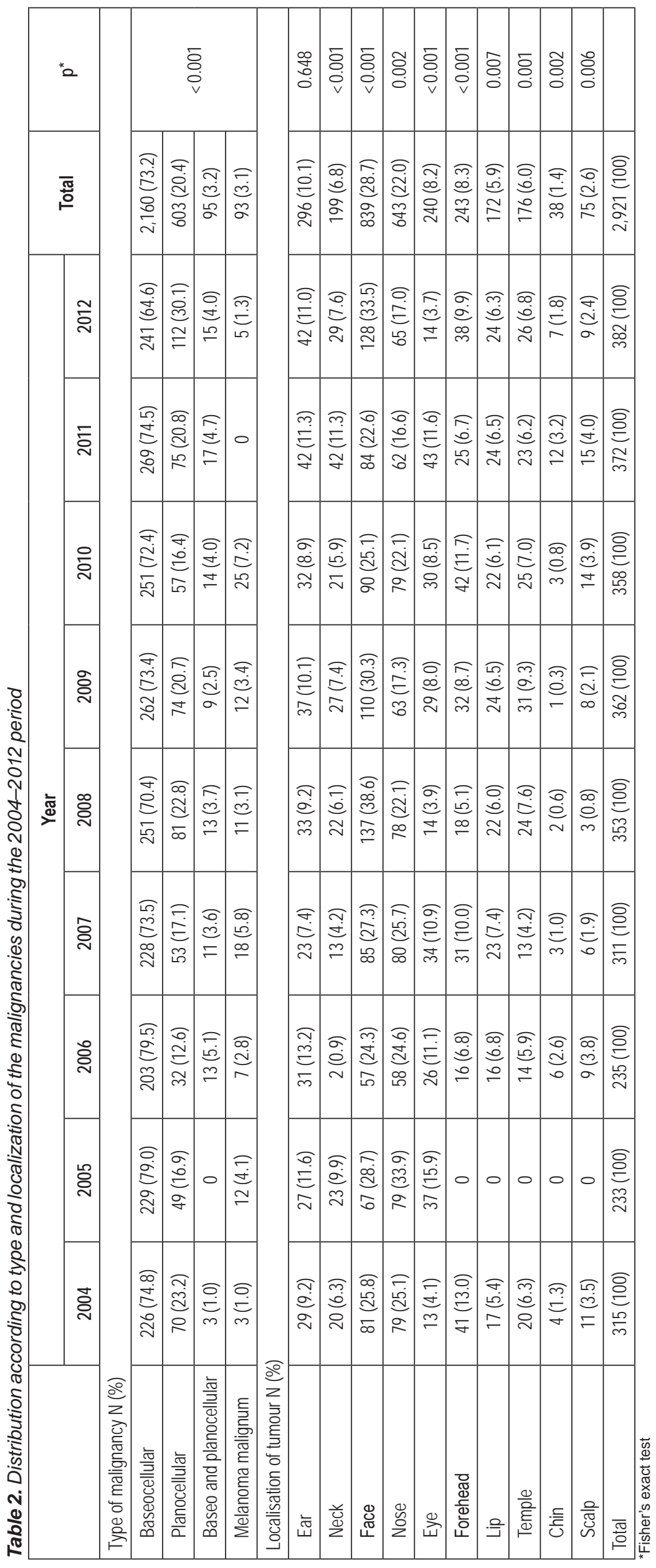


Table 3. Distribution according to type of malignancy, gender, age, settlement, occupation, and workplace

\begin{tabular}{|c|c|c|c|c|c|c|}
\hline & \multicolumn{5}{|c|}{ Type of malignancy $\mathbf{N}(\%)$} & \multirow[b]{2}{*}{$\mathrm{p}$} \\
\hline & Baseocellular & Planocellular & $\begin{array}{c}\text { Basal } \\
\text { and planocellular }\end{array}$ & Melanoma & Total & \\
\hline \multicolumn{7}{|l|}{ Gender } \\
\hline Male & $1,060(49.1)$ & $341(56.6)$ & $41(43.2)$ & $44(47,3)$ & $1,486(50.4)$ & \multirow{2}{*}{$0.005^{*}$} \\
\hline Female & $1,100(50.9)$ & $262(43.4)$ & $54(56.8)$ & $49(52.7)$ & $1,465(49.6)$ & \\
\hline \multicolumn{7}{|l|}{ Age } \\
\hline Median (25-75\%) & $71(63-77)$ & $74(66-80)$ & $76(67-81)$ & $67(57-77)$ & $72(64-78)$ & $<0.001^{\dagger}$ \\
\hline \multicolumn{7}{|l|}{ Place of settlement } \\
\hline Urban & $746(34.6)$ & $190(31.5)$ & $25(26.3)$ & $36(38.7)$ & 997 (33.8) & \multirow{2}{*}{$0.146^{\star}$} \\
\hline Rural & $1,411(65.4)$ & $413(68.5)$ & $70(73.7)$ & $57(61.3)$ & $1,951(66.2)$ & \\
\hline \multicolumn{7}{|l|}{ Workplace } \\
\hline Outdoors & $1,552(71.9)$ & $458(76)$ & $71(75)$ & $61(66)$ & $2,142(72.6)$ & \multirow{2}{*}{$0.089 *$} \\
\hline Indoors & $608(28.1)$ & $145(24)$ & $24(25)$ & $32(34)$ & $809(27.4)$ & \\
\hline \multicolumn{7}{|l|}{ Outdoor professions } \\
\hline Farmers & $656(42.3)$ & $193(7.9)$ & $30(42.2)$ & $31(50.8)$ & $910(42.5)$ & \multirow{4}{*}{$0.905^{*}$} \\
\hline Construction workers & $647(41.6)$ & 2,190 (89.1) & $32(45.1)$ & $22(36.1)$ & 891 (41.6) & \\
\hline Community service & $189(12.2)$ & $62(2.5)$ & $7(9.9)$ & $6(9.8)$ & $264(12.3)$ & \\
\hline Other & $60(3.9)$ & $13(0.5)$ & $2(2.8)$ & $2(3.3)$ & $77(3.6)$ & \\
\hline \multicolumn{7}{|l|}{ Indoor professions } \\
\hline Health and education & $41(6.7)$ & $6(4.1)$ & 0 & $4(12.5)$ & $51(6.3)$ & \multirow{7}{*}{$0.379 *$} \\
\hline Factory worker & $145(23.8)$ & $38(26.2)$ & $10(41.7)$ & $9(28.2)$ & $202(25.1)$ & \\
\hline Service activity & $98(16.1)$ & $23(15.9)$ & $4(16.7)$ & $7(21.9)$ & $132(16.3)$ & \\
\hline Merchants & $50(8.2)$ & $15(10.4)$ & $2(8.3)$ & $1(3.1)$ & $68(8.4)$ & \\
\hline Administration & $144(23.7)$ & $25(17.2)$ & $3(12.4)$ & $8(25.0)$ & $180(22.2)$ & \\
\hline Tourism & $66(10.9)$ & $18(12.4)$ & $1(4.2)$ & $1(3.1)$ & $86(10.6)$ & \\
\hline Others & 64 (10.5) & 20 (13.8) & $4(16.7)$ & $2(6.2)$ & 90 (11.1) & \\
\hline
\end{tabular}

*Fisher's exact test; ${ }^{\dagger}$ Kruskal Wallis test

Table 4. Overall distribution according to type and localization of the malignancy

\begin{tabular}{|c|c|c|c|c|c|c|}
\hline & \multicolumn{5}{|c|}{ Type of malignancy $\mathbf{N}(\%)$} & \multirow[b]{2}{*}{$p^{*}$} \\
\hline & Baseocellular & Planocellular & $\begin{array}{c}\text { Basal } \\
\text { and planocellular }\end{array}$ & Melanoma malignum & Total & \\
\hline \multicolumn{7}{|l|}{ Localisation } \\
\hline Ear & $200(9.7)$ & $79(13.3)$ & $10(9.3)$ & $7(8.0)$ & $296(10.1)$ & 0.039 \\
\hline Neck & $137(6.3)$ & $38(6.4)$ & $10(9.3)$ & $14(15.9)$ & $199(6.8)$ & 0.004 \\
\hline Face & $598(28.1)$ & $176(29.7)$ & $33(30.5)$ & $32(36.4)$ & $839(28.7)$ & 0.236 \\
\hline Nose & $533(25.1)$ & $81(13.8)$ & $20(18.4)$ & $9(10.2)$ & $643(22.1)$ & $<0.001$ \\
\hline Eye & $201(9.4)$ & $25(4.2)$ & $5(4.6)$ & $9(10.2)$ & $240(8.2)$ & $<0.001$ \\
\hline Forehead & $173(8.1)$ & $57(9.5)$ & $11(10.2)$ & $2(2.3)$ & $243(8.3)$ & 0.063 \\
\hline Lip & $82(3.8)$ & $81(13.8)$ & $6(5.6)$ & $2(2.3)$ & $171(5.9)$ & $<0.001$ \\
\hline Temple & $134(6.2)$ & $33(5.6)$ & $6(5.6)$ & $3(3.4)$ & $176(6.0)$ & 0.627 \\
\hline Chin & $25(1.1)$ & $7(1.2)$ & $2(1.9)$ & $4(4.5)$ & $38(1.3)$ & 0.057 \\
\hline Scalp & $49(2.2)$ & $15(2.5)$ & $5(4.6)$ & $6(6.8)$ & $75(2.6)$ & 0.026 \\
\hline Total & $2,132(100)$ & $592(100)$ & $108(100)$ & $88(100)$ & $2,920(100)$ & \\
\hline
\end{tabular}


Table 5. Distribution according to type of malignancy, colour of eyes and hair

\begin{tabular}{|c|c|c|c|c|c|c|}
\hline & \multicolumn{5}{|c|}{ Type of malignancy $\mathrm{N}(\%)$} & \multirow{2}{*}{$\mathrm{p}^{*}$} \\
\hline & Baseocellular & Planocellular & Basal and planocellular & Melanoma malignun & Total & \\
\hline \multicolumn{7}{|c|}{ Hair colour } \\
\hline Red & $1(0.1)$ & 0 & 0 & 0 & $1(0.1)$ & \multirow{4}{*}{0.903} \\
\hline Blond & $395(31.1)$ & $113(32.8)$ & $16(28.1)$ & $12(25.0)$ & $536(31.2)$ & \\
\hline Brown & $789(62.2)$ & $207(60.2)$ & $37(65.0)$ & $30(62.5)$ & $1,063(61.9)$ & \\
\hline Black & $83(6.6)$ & $24(7.0)$ & $4(6.9)$ & $6(12.5)$ & $117(6.8)$ & \\
\hline Total & $1,268(100)$ & $344(100)$ & $57(100)$ & $48(100)$ & $1,717(100)$ & \\
\hline \multicolumn{7}{|c|}{ Eye colour } \\
\hline Blue & $1(0.2)$ & 0 & 0 & 0 & $1(0.1)$ & \multirow{4}{*}{0.379} \\
\hline Green & $160(31.8)$ & $25(22.5)$ & $7(35.0)$ & $10(37.1)$ & $202(30.6)$ & \\
\hline Brown & $304(60.4)$ & $76(68.5)$ & $11(55.0)$ & $12(44.4)$ & $403(61.0)$ & \\
\hline Black & $38(7.6)$ & $10(9.0)$ & $2(10.0)$ & $5(18.5)$ & $55(8.3)$ & \\
\hline Total & $503(100)$ & $111(100)$ & $20(100)$ & 27 (100) & $661(100)$ & \\
\hline
\end{tabular}

*Fisher's exact test

is 5.7 (interquartile range 83.5 to 99.5 hours) in the range of 386 to 279 hours of sunshine during 50 years (Fig. 2). Based on the results from the measuring stations it can be concluded that the duration of sunshine increases.

The number of sunny days in Osijek-Baranja County during the period under consideration was 48.5 (interquartile range from 8.5

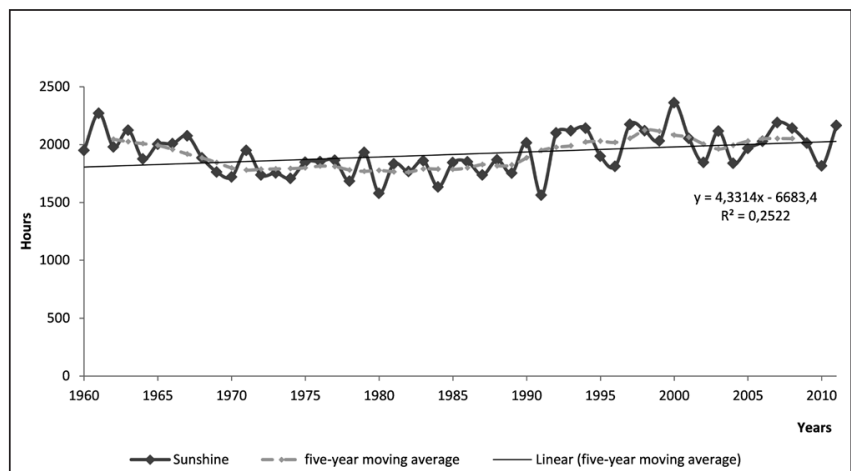

Fig. 1. Time series of sunshine hours with 5-year adjusted average and 50-year (1960-2011) linear trend of sunshine hours.

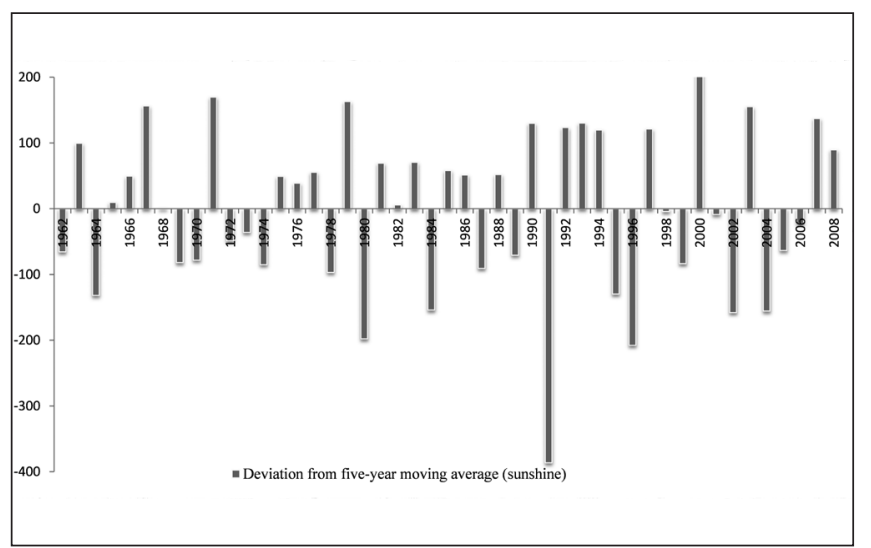

Fig. 2. Deviation from 5-year average of sunshine hours for 50-year period (1960-2011). to 72 days) in the range 0 to 113 days, and the floating five-year average is 60 clear days (interquartile range 5 to 72 days), the range of at least 0 to 85 days (Fig. 3). The mean deviation from the floating five-year average of sunny days is -1 day (interquartile range -10.6 to 7.9 days) ranging from -25 to 32 days. This indicates that the number of clear days almost unchanged. By

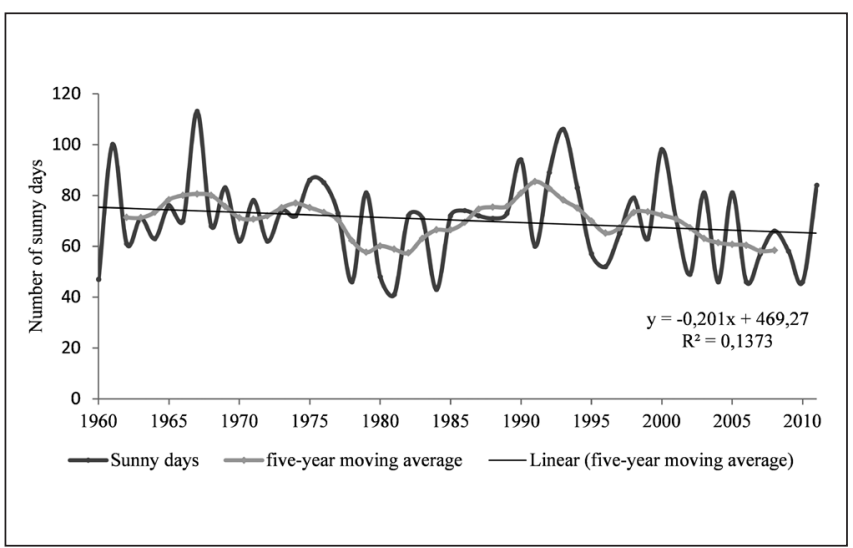

Fig. 3. Time series - number of sunny days compared to adjusted 5 and 50-year (1960-2011) average.

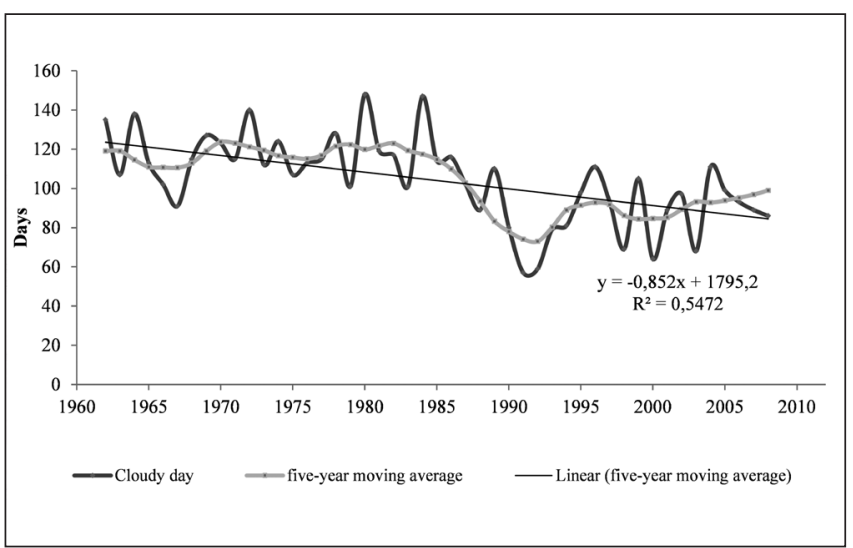

Fig. 4. Time series - number of cloudy days compared to adjusted 5 and 50-year (1960-2011) average. 
analyzing the elements at the climate monitoring station in Osijek from 1961-2011, there is a significant increase in the number of clear days from 1990-1995 and during 2000.

The mean number of cloudy days in Osijek-Baranja County from 1960-2011 is 80 days (interquartile range from 8.5 to 110 days), and the floating five-year average is 85 days (interquartile range 5 to 112 days) with a decreasing trend in the number of cloudy days (Fig. 4). The mean deviation from the floating fiveyear average of cloudy days is -0.8 days (interquartile range -8.7 to 7.3 days) in the range from -25 to 30 days. The average daily cloud cover from 1960-2011 was 51 (interquartile range from 8.5 to 72 ), and the floating five-year average was 62 (interquartile range from 5.3 to 72 days ). The average bias of the floating five-year average is -1.1 (interquartile range -10.4 to 7.9 ) ranging from -25.4 to 32.4 , mean cloudiness was 5.5 (interquartile range 5-6.25), ranging from 0 to 84, while the five-year floating mean cloudiness in Osijek-Baranja County was 5.4 (interquartile range $5-5.8$ ) in the range 0 to 65 . All results of this study show that in Osijek-Baranja County in the last 50 years there has been a downward trend in cloudiness and upward trend in the mean annual sum of sunshine, thereby increasing the annual radiant solar energy.

Table 6. Correlation between sun hours and number of patients regarding gender, place of settlement, localization, and type of malignancy

\begin{tabular}{|c|c|c|}
\hline & Spearman's rho & $p$ \\
\hline \multicolumn{3}{|l|}{ Gender } \\
\hline Male & 0.226 & 0.559 \\
\hline Female & 0.317 & 0.406 \\
\hline Total & 0.083 & 0.831 \\
\hline \multicolumn{3}{|l|}{ Settlemet - urban } \\
\hline Male & -0.664 & 0.051 \\
\hline Female & 0.486 & 0.187 \\
\hline \multicolumn{3}{|l|}{ Settlement - rural } \\
\hline Male & 0.100 & 0.797 \\
\hline Female & 0.183 & 0.637 \\
\hline \multicolumn{3}{|l|}{ Age } \\
\hline$\leq 40$ & -0.475 & 0.197 \\
\hline $41-55$ & -0.458 & 0.215 \\
\hline $56-70$ & 0.159 & 0.683 \\
\hline $71-85$ & 0.300 & 0.433 \\
\hline$>85$ & 0.076 & 0.847 \\
\hline \multicolumn{3}{|l|}{ Type of malignancy } \\
\hline Planocellular & -0.360 & 0.342 \\
\hline Baseocellular & 0.500 & 0.170 \\
\hline Plano and baseocellular & -0.050 & 0.898 \\
\hline Melanoma & 0.695 & 0.038 \\
\hline \multicolumn{3}{|l|}{ Most frequent localization } \\
\hline Face & 0.075 & 0.847 \\
\hline Nose & 0.117 & 0.765 \\
\hline Ear & -0.193 & 0.618 \\
\hline Forehead & 0.067 & 0.864 \\
\hline
\end{tabular}

Using the Spearman's correlation coefficient $(\rho)$ the association between the number of patients with malignant neoplasms of the skin, head and neck by gender, place of residence, age, location and type of malignant neoplasms, and the number of hours of sunshine was assessed. It revealed that the increase in the hours of sunshine leads to a greater number of patients with malignant melanoma as one type of malignant neoplasms $(\rho=0.695, p=0.038)$, while other weaker correlation is not significant (Table 6).

\section{DISCUSSION}

This was the first study on the incidence and characteristics of malignant neoplasms of the head and neck in Osijek-Baranja County ever conducted. The study included the period from 2004-2012 because there were well documented credible data for all patients with cutaneous neoplasia whose biopsies were analyzed at the Department of Pathology, Clinical Hospital Osijek. As stated above, all or nearly all patients in Osijek-Baranja County were treated at the hospital in Osijek, which enabled the estimation of frequency and assessment of trends of disorders in the reporting period. The data on morbidity were compared with the data of climatic elements (values of mean monthly and annual cloudiness, values of mean, monthly and annual sum of sunshine) for 50 years (1961-2011) for the measuring area of the city of Osijek.

The average annual incidence for the monitored period was approximately 104/100,000 in the range of about 91/100,000 in 2004 to about 123/100,000 in 2012. In the initial years of the research there were 255-302 cases per year, and in the final years of the research there were from 347-372 cases annually. Moreover, according to the census the county had over 330,000 inhabitants in 2001, while in 2011 had only 305,000 inhabitants (a decrease in population for over 25,000 ) and therefore the relative proportion of patients increased.

Although, according to population census in 2001 and 2011 men were less represented (48.2\%) than women, men more often suffer from cancer (50.4\% vs. $49.6 \%$ ). In addition, squamous cell carcinoma occurred more frequently in men than in women (56.6\% vs. 43.4\%), but there was no difference regarding gender in the incidence of the much more frequent basal cell carcinoma, which represented $73.2 \%$ of all cases. It was also found that significantly younger patients suffer more from malignant melanoma, the share was on average $3.1 \%$, with a mean age of 69 years (interquartile range $60-75$ years), as compared to the overall mean age of the patients which was 72 years (interquartile range 64-78 years). The patients were mostly from rural areas, 1,952 (66.2\%) with no significant difference during the studied years; 2,137 (72.4\%) of the patients worked outdoors and 813 (27.6\%) worked indoors. Regarding outdoor occupations, 907 of them were farmers (42.4\%) and 889 construction workers (41.6\%), whereas indoor occupations included 204 factory workers (25.1\%), with significant differences according to age. However, no significant differences between the type of malignant neoplasms and the place of residence, workplace and indoors or outdoors occupations were found.

The face is the most common localisation of malignant neoplasms in 839 (28.7\%) and nose in 643 patients (22.0\%). Regarding location, the ear and lip had significantly more squamous cell 
malignancies, on the neck, eye and head malignant melanoma was more frequent, whereas basal cell neoplasms were found on the nose.

Information about hair colour and eyes had not been recorded, but was subsequently collected. For 1,717 patients data on hair colour were collected and for 616 of them data on eye colour as well, but no statistically significant differences in the type and frequency of malignant parameters were established.

Based on the data, the mean duration of sunshine during 50 years was 1,737 hours per year, and the secondary floating five-year average was 1,781 hours of sunshine. The duration of sunshine (insolation) during 50 years at the measuring station of the city of Osijek indicates that in the period from 1992-2011 there was repeated increase in insolation value of over 2,000 hours per year. The linear floating five-year average shows an increasing trend. Similar data were found in the IUU Climate Atlas of Croatia published in 2008. The total annual duration of sunshine shows that Croatia is a very sunny country, with the Croatian coast being one of the sunniest places in Europe. Along the Adriatic coast the annual duration of sunshine is more than 2,000 hours (between 5.9 hours per day in Rijeka to 7.4 hours per day in the island Hvar). Going from the sea to land the number of hours of sunshine decreases. The characteristics of the distribution of mean annual sunshine duration in the lowland part of the continental Croatia show mild increase in value from west to east and north. The values range from 1,825 hours per year in Slavonski Brod (5 hours per day) to 2,044 hours per year in Đurđevac (5.6 hours per day). Relative insolation from 1971-2000 was between 0.5 and 2.1\% higher than from 1961-1990 (5).

The Fifth National Communication of the Republic of Croatia under the United Nations Framework Convention on Climate Change (UNFCCC) in November 2009 concluded that the consequence of the rapid warming of the atmosphere in the last period was the warmest decade since the beginning of the 20th century. From the year 2000, the seven warmest years were recorded in Zagreb, six in Gospic and Crikvenica, five in Hvar and four in Osijek. From the data of Croatian measuring stations as well as from data of other monitoring stations it can be concluded that in continental Croatia relative duration of sunshine increases.

The number of sunny days in Osijek-Baranja County during the study period was 48.5 in the range from 0 to 113 days, and the floating five-year average for clear days was 60 , ranging from a minimum of $0-85$ days. This indicates that the number of clear days did not change. By analyzing the elements of the climate monitoring station in Osijek from 1961-2011, a significant increase in the number of clear days from 1990-1995 and during 2000 could be observed.

Throughout the years, the number of sunny days in autumn has been reduced while the number of cloudy days has increased. Cloudiness is a very important element of the climate as a direct participant in the creation of relations between radiation and the Earth's atmosphere. Cloudiness is the amount of clouds covering the sky. It is estimated visually in parts of the sky blocked by clouds. This quantity is expressed in tenths of sky (0/10), a completely clear sky is shown with zero tenths, while overcast with ten tenths $(10 / 10)(5,6)$. The mean number of cloudy days in Osijek-Baranja County from 1960-2011 was 80 days and the floating five-year average was 85 days with a decreasing trend in the number of cloudy days. For example, during 1970 in Osijek there were about 120 cloudy days versus 90 cloudy days in 2011 . These data match the data from the Climatic Atlas of Croatia published in 2008 (5).

The characteristics of cloudiness are closely associated with the geographical position of Croatia, ranging from the Pannonian Basin, over the Dinara mountain range to the east coast. The average annual cloudiness is generally between $4 / 10$ and $7 / 10$. The difference in cloudiness of 3/10 indicates a large diversity in a relatively small area of Croatia. Generally, Croatia can be divided into a cloudier part with the annual cloudiness greater than 5/10 and the brighter part where less than half of the sky is overcasted. Cloudy days with daily average cloudiness of $8 / 10$ are observed in the mountain region where the year has about 35\% to $40 \%$ cloudy days (approximately 120-140 days). In the inlands the number of cloudy days is smaller, about $30 \%$ in the east, up to $35 \%$ in central Croatia. In the lowland areas of Croatia cloudiness increases from the eastern to north-western and central part of Croatia. In the most eastern part of Croatia mean annual cloudiness is 5.5/10, while in the central part of Croatia it is between 6 and 6.5/10. Regarding the seasons, in the period 1961-1990 in the cold part of the year cloudiness and the number of cloudy days was reduced as compared to an increased number of clear days. From 1971-2000, cloudiness decreased in most parts of Croatia as compared to the previous reference period, and the number of cloudy days decreased accordingly (5). All these meteorological data for Osijek-Baranja County in the past 50 years confirm a declining trend of cloudiness and upward trend of the mean annual sum of sunshine which results in increase in annual radiant solar energy.

In this regard, the increase in hours of sunshine in OsijekBaranja County led to a significantly higher morbidity of malignant melanoma $(\rho=0.695, p=0.038)$, while this relationship was not established for other types of neoplasms.

Overall, skin cancers are the most common tumours in humans and they have been occuring with increasing frequency in recent decades, which is consistent with our results. It is, among other things, linked with exposure to UV radiation, either by direct exposure to sun for professional or recreational reasons or artificial sources via tanning, phototherapy etc. A higher degree of awareness of dangers of exposure to UV radiation is considered the major reason for the decrease in the number of cases in areas where protective and preventive measures and activities were taken (10-12). One of the main economic activities in OsijekBaranja County is agriculture. In the last 15 to 20 years, construction had a significant proportion among all economic sectors, in both the whole country and in this county in particular, due to the reconstruction of war devastated houses, road reconstruction and similar activities. Therefore, it is no surprise that a large proportion of the patients are construction workers working outdoors and people living in rural areas.

In accordance with other studies, it has been confirmed that the malignant neoplasms of the skin, especially on the uncovered parts, are diseases of the elderly and they are the result of decades of long-term incubation and cumulative effect of exposure to risk factors, among which direct sun exposure seems to play an important role $(10,11,18,19)$.

Like most authors, we have also found that baselocelluar carcinoma is the most common malignant tumour of the skin which represents between $73 \%$ and $76 \%$ of all tumours, although there 
were differences in the overall incidence and gender since some authors state higher frequency in women and others in men, but in our research this was not the case (20).

Subsequent data collection of the hair and eyes colour unfortunately has not brought the expected contribution, as opposed to a similar study conducted in Split-Dalmatia County, which has proven a greater risk of developing malignant skin diseases in people with fair hair and light-coloured eyes $(10,12)$.

The specificity of our research lies in the fact that significantly more frequent occurrence of squamous cell tumours in males than in females was observed for which we do not have a rational explanation, but there was only one finding from this research according to which men are more prone to develop non-melanoma tumours. Data from our study show that, on average, younger patients were more frequently diagnosed with malignant melanoma. An increase in the hours of sunshine led to a greater number of patients with malignant melanoma, but not with other types of tumours. However, it was also found that malignant melanoma has a share of only $3.1 \%$ of all tumours. The above facts show that the sun has higher significance in formation of this type of tumour, thus further research is needed.

An increased incidence of skin and neck tumours during the monitored period in Osijek-Baranja County is surprising together with a relatively high incidence of tumours in total, which exceeds the present knowledge taking into account the fact that only cutaneous head and neck tumours, which account for only $9 \%$ of the skin surface, were analysed. We determined the approximate incidence ranging from about 91/100,000 in 2004 to about 123/100,000 in 2012, with increasing trend. For example, according to IARC the age-standardized incidence of melanoma in all locations of Croatia is estimated to be about $7 / 100,000$ per year, and they account for between $3 \%$ and $11 \%$ of all tumours (3.1\% in our study), while for other tumours there are no IARC data available for Croatia (20).

According to some authors, the incidence of skin cancer of the head and neck varies from $55.1 \%$ to $95.5 \%$ of all skin tumours. With regard to this, a rough calculation shows that the total annual incidence of skin cancer in all locations in Croatia, based on the determined frequency and share of certain types of tumours in Osijek-Baranja County (only skin tumors of the head and neck) could be between 155 and 230/100,000 or even higher, which is significantly more than the estimation of nonmelanoma in Croatia with the annual incidence of 83.7/100,000 in men and 81.9/100,000 in women. Even if we add the average share of about $10 \%$ of melanoma, it is clear that the overall frequency of skin tumours in Croatia is underestimated $(9,10)$. This frequency is comparable to some parts of Brazil (Goiania), where the recorded annual standardized incidence of all skin cancers is around 220/100,000, and is much higher than in Ireland (130/100,000), some parts of Italy (100/100,000), Germany (96/100,000), our neighbouring country Slovenia $(60 / 100,000)$, and Slovakia $(50 / 100,000)(25,33)$, whereas the highest incidence, according to some authors, is found in Australia (500 to $1,600 / 100,000$ in some regions) and the southern parts of the USA (300/100,000) (20-23).

However, this study has its weaknesses. As already mentioned, the data on hair and eye colour were not collected for all patients, as well as the data on family history (other cases of illness in the family) and potential chromosomal aberations. The data on exposure to other potential risk factors such as arsenic, polycyclic hydrocarbons, monitored and treated moles, previous skin tumours, organ transplantation, immunosuppressive therapy, HPV infection, etc. are also missing (24-26). Therefore, a more comprehensive research has to be conducted in the future.

\section{CONCLUSIONS}

The last 50 years in Osijek-Baranja County have seen a declining trend in the number of cloudy days and upward trend in the mean annual sum of sunny days. When compared, it is obvious that the increase in sunny days results in a higher number of patients suffering from malignant melanoma $(\rho=0.695, p=0.038)$.

The malignant neoplasms of the skin, head and neck occur after decades of exposure and as a cumulative effect of exposure to risk factors. A direct exposure to sun seems to play an important role, especially with regard to melanoma.

It can also be concluded, that in the absence of reliable and credible data on skin tumours in the Republic of Croatia, especially on those on the exposed areas of skin, this research represents a good base for future research.

\section{Conflict of Interests}

None declared

\section{REFERENCES}

1. Lucas R, McMichael T, Smith W, Armstrong B. Solar ultraviolet radiation: global burden of disease from solar ultraviolet radiation. Environmental burden of disease series, no. 13. Geneva: WHO; 2006.

2. Diffey BL. Stratospheric ozone depletion and the risk of nonmelanoma skin cancer in a British population. Phys Med Biol. 1992 Dec;37(12):2267-79.

3. Vanicek K, Frei T, Litynska Z, Schmalwieser A. UV-Index for the public: a guide for publication and interpretation of solar UV Index forecasts for the public prepared by the Working Group 4 of the COST-713 Action „UVB Forecasting“. COST-713 Action. Brussels; 1999.

4. United States Environmantal Protection Agency. Community-based ultraviolet radiation (UV) risk education: the SunWise Program handbook [Internet]. Cincinnati: U.S. EPA; 2002 [cited 2014 Aug 10]. Available from: http://nepis.epa.gov/Adobe/PDF/30004IQV.pdf.

5. Zaninović K, editor. Climate atlas of Croatia 1961-1990, 1971-2000. Zagreb: Meteorological and Hydrological Service of Croatia; 2008.

6. Chaillol I, Boniol M, Middleton R, Doré JF, Autier P, Gavin A. Seasonality of cutaneous melanoma diagnosis in Northern Ireland with a review. Melanoma Res. 2011 Apr;21(2):144-51.

7. Stanton WR, Janda M, Baade PD, Anderson P. Primary prevention of skin cancer: a review of sun protection in Australia and internationally. Health Promot Int. 2004 Sep;19(3):369-78.

8. Walker D. Cloud effects on erythemal UV radiation in a complex topography. Veröffentlichungen/MeteoSchweiz. 2010;(86):1-106.

9. Strnad M, Lipozenčić J. Epidemiology of skin tumours in world and Croatia. In: Lipozenčić J, Pašić A, Strnad M, Puizina-Ivić N, Ručević I, Barišić-Druško V, et al. Dermatologic oncology. Zagreb: Medicinska naklada; 2009. (In Croatian.)

10. Celić D, Lipozenčić J, Jurakić Tončić R, Ledić-Drvar D, Marasović D, Puizina-Ivić N, et al. The incidence of basal cell carcinoma in Croatia: an epidemiological study. Acta Dermatovenerol Croat. 2009;17(2):108-12.

11. American Cancer Society. Cancer facts \& figures 2014. Atlanta: American Cancer Society; 2014.

12. Marasović D. Incidence of skin tumours in different geographical areas [dissertation]. Split: University of Split, School of Medicine; 1996. (In Croatian.) 
13. Gandini S, Sera F, Cattaruzza MS, Pasquini P, Picconi O, Boyle P, et al. Meta-analysis of risk factors for cutaneous melanoma: II. Sun exposure. Eur J Cancer. 2005 Jan;41(1):45-60.

14. Braun MM, Tucker MA, Devesa SS, Hoover RN. Seasonal variation in frequency of diagnosis of cutaneous malignant melanoma. Melanoma Res. 1994 Aug;4(4):235-41.

15. Sun EC, Fears TR, Goedert JJ. Epidemiology of squamous cell conjunctival cancer. Cancer Epidemiol Biomarkers Prev. 1997 Feb;6(2):73-7.

16. Republic of Croatia. Croatian Bureau of Statistics. Census 2001. Census of population, households and dwellings, 31st March 2001 [Internet]. Zagreb: CBS; 2001 [cited 2015 Dec 31]. Available from: http://www. dzs.hr/default_e.htm.

17. Republic of Croatia. Croatian Bureau of Statistics. Census of population, households and dwellings 2011 [Internet]. Zagreb: CBS; 2011 [cited 2015 Dec 31]. Available from: http://www.dzs.hr/default_e.htm.

18. Moehrle M, Heinrich L, Schmid A, Garbe C. Extreme UV exposure of professional cyclists. Dermatology. 2000;201(1):44-5.

19. Schwartzbaum J, Ahlbom A, Feychting M. Cohort study of cancer risk among male and female shift workers. Scand J Work Environ Health. 2007 Oct;33(5):336-43.

20. World Health Organization; International Agency for Research of Cancer. Cancer incidence in five continents. Volume X. Lyon: IARC; 2014.
21. Rogers HW, Weinstock MA, Harris AR, Hinckley MR, Feldman SR, Fleischer AB, et al. Incidence estimate of nonmelanoma skin cancer in the United States, 2006. Arch Dermatol. 2010 Mar;146(3):283-7.

22. Van der Pols JC. Epidemiology of basal cell and squamous cell carcinoma of rhe skin. In: Dummer R, Pittelkow MR, Iwatsuki K, Green A, Elwan NM, editors. Skin cancer - a world-wide perspective. Heidelberg: Springer; 2011.

23. Minelli L. Statistical survey of basal cell carcinoma in Londrina (Paraná, Brazil). An Bras Dermatol. 1987;62:321-5. (In Portuguese.)

24. Lear JT, Harvey I, de Berker D, Strange RC, Fryer AA. Basal cell carcinoma. J R Soc Med. 1998 Nov;91(11):585-8.

25. Freire-Maia N, Cavalli IJ. Albinism, skin carcinoma and chromosome aberrations. Clin Genet. 1980 Jan;17(1):46-7.

26. Gasparro FP. The role of PUVA in the treatment of psoriasis. Photobiology issues related to skin cancer incidence. Am J Clin Dermatol. 2000 Nov-Dec;1(6):337-48.

Received August 20, 2014

Accepted in revised form April 6, 2015 